\title{
Unpreventable Maternal Death In Spite Of the Advances in Antenatal Care; a Case Report
}

\author{
Warushahennadi $\mathbf{J}^{*}$ \\ Department of Forensic Medicine, Faculty of Medicine, University of Ruhuna, Sri Lanka.
}

\begin{abstract}
Aortic dissection (AoD) is a very rare but potentially fatal entity during pregnancy. The incidence of acute aortic dissection during pregnancy is about 0.4 cases per 100000 persons yearly. A 28 -year-old 38-week primi was referred to the hospital following sudden onset of burning epigastric pain which developed while returning from the routine visit to the cardiologist. She was treated for gastritis in the ward and a few hours later she developed shortness of breath, tight chest pain and tachycardia. Emergency LSCS was done and a live baby was delivered and she had sudden cardiac arrest soon after the extubation, in spite of the intensive resuscitation measures including six units of blood transfusion. She was diagnosed with mild aortic regurgitation and trivial mitral regurgitation before the pregnancy and was referred to the cardiologist during the first trimester and was confirmed with low cardiac risk. She was seen by the cardiologist during all 3 trimesters and was concluded with low cardiac risk. The autopsy revealed $2750 \mathrm{ml}$ of blood in the chest cavity and $750 \mathrm{ml}$ of blood in the abdominal cavity. There was a collection of blood which was infiltrated into the mediastinal tissues around the aorta. There were macroscopic features of aortic dissection and the microscopy of the aorta revealed degeneration of tunica media, a through and through tear on intima with leakage of blood into the surrounding tissues. This case report illustrates that the pregnancy itself is a risk factor for the development of AoD which leads to the catastrophic disorder in pregnancy.
\end{abstract}

Keywords: Aortic dissection, Maternal death, Risk factors

Received: 27 April 2019, Revised version accepted: 05 December 2019, Published: 31 December 2019. *Corresponding author: Warushahennadi J, $\triangle$ Email: janakiwh@gmail.com, (iD https://orcid.org/0000-0002-6880-5513

Cite this article as: Warushahennadi J. Unpreventable Maternal Death In Spite Of the Advances in Antenatal Care; a Case Report. Medico-Legal Journal of Sri Lanka, 2019;7(2): 65-67. DOI: http://doi.org/10.4038/mljsl.v7i2.7384

Copyright: @ 2019 with the Medico-legal Journal of Sri Lanka.

This is an open-access article distributed under the terms of the Creative Commons Attribution 4.0 International License, which permits unrestricted use, distribution and reproduction in any medium provided the original author and source are credited.

\section{Introduction}

The incidence of aortic dissection (AoD) during pregnancy is about 0.4 cases per 100000 persons yearly. ${ }^{[1]}$ Although it is a rare entity, the aortic dissection in pregnancy may result in adverse maternal and fetal outcomes. Half of the aortic dissections occur in the third trimester of pregnancy and in the postpartum period when the haemodynamic stress is at the peak. ${ }^{[2]}$ Atherosclerosis, hypertension, genetic disorders of connective tissue such as Marfan`s syndrome, genetic conditions such as turners syndrome, valvular abnormalities and vascular diseases like preeclampsia and Cocaine abuse are some of the risk factors for AoD. The pregnancy alone with no underlying pre-existing risk factors is a risk factor for AoD. The hyper-dynamic changes in pregnancy and the increased oestrogen level which suppresses the synthesis of collagen and elastin may weaken the vascular wall thereby playing an important role in the development of AoD during pregnancy. ${ }^{[3]}$
Although it is difficult to diagnose the AoD, the measurement of diameter of the root of the aorta is a diagnostic criterion of the risk of acute AoD. The aortic root diameter more than $45 \mathrm{~mm}$ in pregnancy has a high risk for AoD. ${ }^{[4]}$

\section{Case report}

A 28-year-old 38-week primi was referred to a tertiary care hospital following sudden onset of burning epigastric pain which developed while returning from the routine visit to the cardiologist. She was treated for gastritis in the ward and a few hours later she developed shortness of breath, tight chest pain and tachycardia. Emergency Lower Segment Caesarean Section (LSCS) was done and a live baby was delivered and she had sudden cardiac arrest soon after the extubation, in spite of the intensive resuscitation measures including six units of blood transfusion. 
She was diagnosed with mild aortic regurgitation (AR) and trivial mitral regurgitation (MR) before the pregnancy and was referred to the Cardiologist during the first trimester and was confirmed with low cardiac risk. She was seen by the Cardiologist during all 3 trimesters and was concluded with low cardiac risk. During the last visit to the Cardiologist which was 30 minutes before admission to the hospital, 2D ECHO revealed low cardiac risk.

The autopsy revealed $2750 \mathrm{ml}$ of blood in the combined chest cavity and $750 \mathrm{ml}$ of blood in the abdominal cavity. There was a collection of blood which was infiltrated into the mediastinal tissues around aorta (Figure 01).

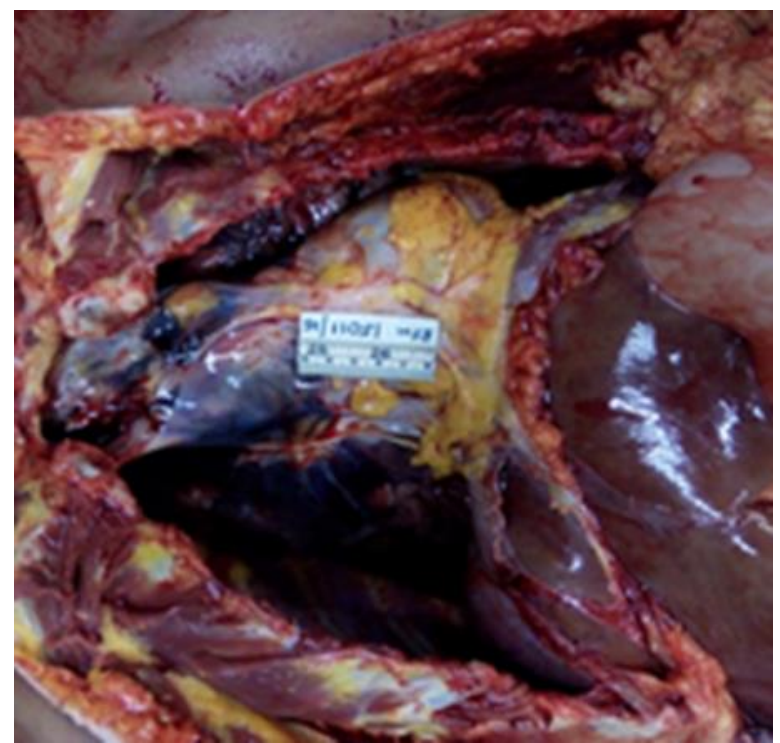

Figure 01: Blood in the chest cavity

The collection of blood was present in the intima of the aorta starting from the route of the aorta extending up to the abdominal aorta above the Ostia of the renal arteries (Figure 02). The exact place of the dissection was unable to be determined at the autopsy.

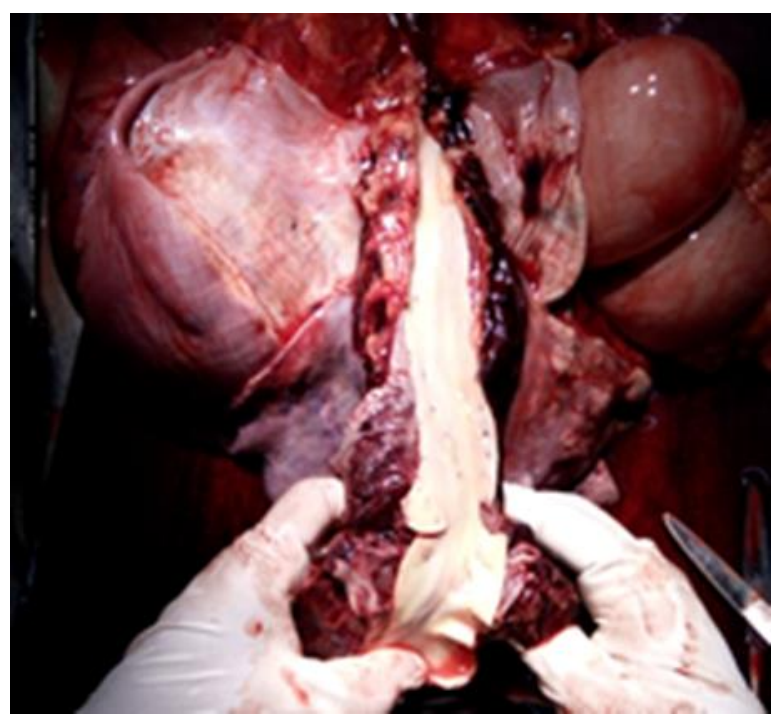

Figure 02: No macroscopical tears on the aorta. Blood in the soft tissues around the aorta is seen.

The abdominal cavity was normal except the postpartum appearance of the sectioned uterus. Several sections obtained from different sites of the aorta for histopathology revealed degeneration of Tunica media and through and through micro tears with leakage of blood into the surrounding tissues of the aorta (Photomicrograph 01).

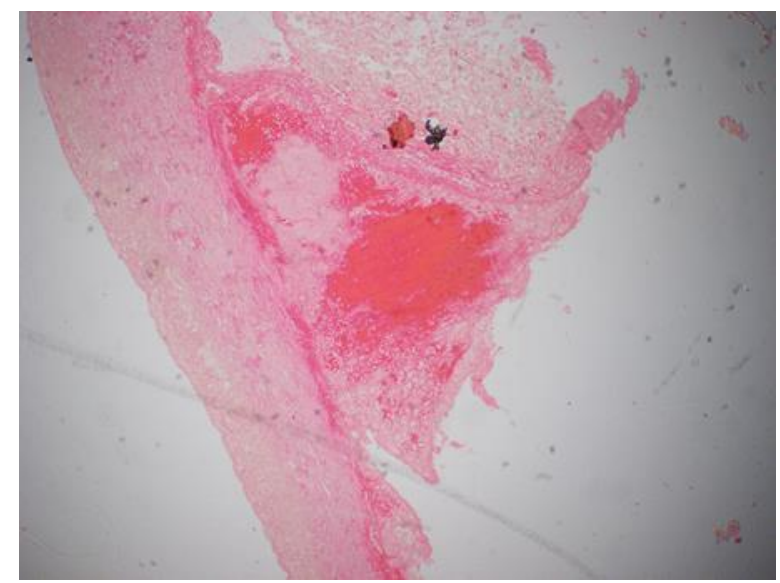

Photomicrograph 01: The aorta revealed degeneration of Tunica media and through and through micro tears with leakage of blood

The cause of death was concluded as haemorrhage and shock following acute aortic dissection in a pregnant woman.

\section{Discussion}

The patient was diagnosed with AR and trivial MR which are not considered as a risk factor for the aortic dissection. According to the available literature, among the valvular heart diseases, the bicuspid aortic 
valve is a risk factor for acute aortic dissection ${ }^{[4]}$ which indicates that the deceased did not have a known preexisting risk factor for the acute aortic dissection. But the hormonal changes during pregnancy which suppresses the synthesis of collagen and elastin weakening the blood vessel walls and the fact that the aortic compression due to gravid uterus increasing the outflow resistance on the aorta are known risk factor for AoD. Therefore, these alterations during the pregnancy may have caused the aortic dissection in the deceased.

The common symptoms at the onset of the AoD are the chest pain accounting for $12 \%$ and the back pain accounting for $55 \%{ }^{[5]}$ but the patient had burning epigastric pain suggesting gastritis. The determination of the low cardiac risk by the cardiologist few hours before may have influenced to take a longer time to suspect AoD.

The patient was evaluated by a cardiologist few hours before death and confirmed low cardiac risk based on the measurements of the root of the aorta which measured less than $40 \mathrm{~mm}$. Thirty minutes later she developed symptoms and was admitted to the hospital. The doctors have suspected AoD and emergency LSCS was done. This implies the catastrophic situation which may arise in similar clinical circumstances.

\section{Conclusions}

Pregnancy itself is a risk factor for the development of AoD which leads to the catastrophic disorder in pregnancy. Therefore the increased risk of AoD during pregnancy should be kept in mind in patients with symptoms even without pre-existing risk factors.

\section{Disclosure statement}

Conflicts of interests: The authors declare that they have no conflicts of interests.

Funding: None

\section{References}

1. Thalmann $\mathrm{M}$, Sodeck $\mathrm{GH}$, Domanovits $\mathrm{H}$, Gassberger M, Loewe C, et al. Acute type A aortic dissection and pregnancy: a popular - based study. Eur. J Cardio thorac Surg 2011; 39(6):15963.

2. Ozdemir B. Aortic Dissection in Pregnancy. Turkish Clinics J Int Med Sci 2005;1(50):54-8.

3. Shi-MinYuan. Aortic Dissection During Pregnancy: A Difficult Clinical Scenario: Review. Clini. Cardiol. 2013;36(10):576-84

4. Nishadi AA, R Herath, G R C Silva, R Mohomead, P Wijesinghe, S Padumadasa et al. A fatal case of dissecting aortic root aneurysm due to undiagnosed Marfan syndrome in the puerperium. Sri Lanka Journal of Obstetrics and Gynaecology. 2012;34(2):55-7. DOI: http://dx. doi.org/10.4038/ sljog.v34i2.4829

5. Fuster V, Andrews P. Medical treatment of the aorta. L. Cardiol Clin. 1999; 17(4) 697-715 